\title{
Cirrhosis and Gastrointestinal Bleeding
}

\author{
Shih-Wei Lai $\cdot$ Kuan-Fu Liao
}

Received: 19 September 2012/ Accepted: 26 September 2012/Published online: 21 October 2012

(C) Springer Science+Business Media New York 2012

To the Editor,

We read the article by Kalafateli et al. [1], which assesses the relationship between non-variceal gastrointestinal bleeding and cirrhosis, with great interest. The authors comment that the related data of this issue are scanty. In fact, two cohort studies found by utilizing the Taiwan National Health Insurance database have shown that cirrhotic patients had an increased risk of peptic ulcer bleeding (hazard ratio range, 3.19-4.22) [2,3]. These two studies with meaningful findings have added to the evidence on this issue.

To date, because of no specific treatment for cirrhosis, the risk of cirrhosis-induced gastrointestinal bleeding could not be well controlled. From the view of preventive medicine, prevention of hepatitis $\mathrm{B}$ and $\mathrm{C}$ virus infections for prevention of cirrhosis should be the more practical policy to effectively reduce the risk of cirrhosis-related

\section{S.-W. Lai}

School of Medicine, China Medical University, Taichung 404, Taiwan

S.-W. Lai

Department of Family Medicine, China Medical University Hospital, Taichung 404, Taiwan

K.-F. Liao ( $\square)$

Department of Internal Medicine, Taichung Tzu Chi General Hospital, No.66, Sec. 1, Fongsing Rd., Tanzi, Taichung 427, Taiwan

e-mail: kuanfuliao@yahoo.com.tw

K.-F. Liao

Graduate Institute of Integrated Medicine, China Medical

University, Taichung 404, Taiwan

K.-F. Liao

Department of Health Care Administration, Central Taiwan University of Science and Technology, Taichung 406, Taiwan gastrointestinal bleeding [4]. Therefore, hepatitis B vaccination, interrupting the transmission routes of hepatitis $\mathrm{C}$ infection, screening for blood donors with hepatitis $\mathrm{C}$ antibody, treatment with interferon and ribavirin for hepatitis $\mathrm{C}$ infection, and reduction in alcohol intake, should be diligently implemented in clinical practice [5].

\section{Conflict of interest None.}

\section{References}

1. Kalafateli M, Triantos CK, Nikolopoulou V, Burroughs A. Nonvariceal gastrointestinal bleeding in patients with liver cirrhosis: a review. Dig Dis Sci. 2012 (Epub ahead of print). doi:10.1007/ s10620-012-2229-x.

2. Hsu YC, Lin JT, Chen TT, Wu MS, Wu CY. Long-term risk of recurrent peptic ulcer bleeding in patients with liver cirrhosis: a 10-year nationwide cohort study. Hepatology. 2012;56:698-705.

3. Luo JC, Leu HB, Hou MC, et al. Cirrhotic patients at increased risk of peptic ulcer bleeding: a nationwide population-based cohort study. Aliment Pharmacol Ther. 2012;36:542-550.

4. Sung JL. Prevention of hepatitis B and C virus infection for prevention of cirrhosis and hepatocellular carcinoma. J Gastroenterol Hepatol. 1997;12:S370-S376.

5. Chen DS. Hepatocellular carcinoma in Taiwan. Hepatol Res. 2007; 37:S101-S105. 